\title{
IC 5217 as a double-shell, point-symmetric planetary nebula with a very narrow waist
}

\author{
L. F. Miranda ${ }^{1}$, S. Ayala ${ }^{1,2}$, R. Vázquez ${ }^{2}$, and P. F. Guillén ${ }^{2}$ \\ 1 Instituto de Astrofísica de Andalucía (CSIC), Camino Bajo de Huétor 50, 18008 Granada, Spain \\ e-mail: [lfm;sayala]@iaa.es \\ 2 Instituto de Astronomía, UNAM, Ap. Postal 877, 22800 Ensenada BC, Mexico \\ e-mail: [vazquez; sayala; fguillen]@astrosen. unam.mx
}

Received 4 May 2006 / Accepted 9 June 2006

\begin{abstract}
Aims. Identification of the structural components and analysis of the internal kinematics in the planetary nebula IC 5217. Methods. Narrow-band images and high resolution long-slit spectra in the $\mathrm{H} \alpha,[\mathrm{N}$ II] and [O III] emission lines, and VLA $6 \mathrm{~cm}$ continuum data.

Results. IC 5217 is composed of a very bright equatorial ring, open bipolar lobes, off-axis point-symmetric features, on-axis distant faint regions, and an off-axis very elongated bipolar structure. The ring, open lobes, point-symmetric features and distant faint regions appear as elements of a single point-symmetric bipolar shell with a very narrow waist. This shell presents an axis ratio of $\sim 37$ and an aspect ratio of $\sim 5$. The $6 \mathrm{~cm}$ data show that the ring is an extremely flat disk with a central hole. Expansion velocity in the ring ranges from $\leq 10 \mathrm{~km} \mathrm{~s}^{-1}$ in He II up to $\simeq 27 \mathrm{~km} \mathrm{~s}^{-1}$ in [N II], whereas a velocity of $\sim 460 \mathrm{~km} \mathrm{~s}^{-1}$ is estimated for the polar regions of the bipolar shell. Strong acceleration of the outer regions of the ring is observed. The elongated bipolar structure probably represents a highly collimated (aspect ratio 12), high velocity cylindrical-like shell. A collimated agent (wind or jet) would account for the shaping of the bipolar shell if this agent has operated in the direction perpendicular to the equatorial disk. The point-symmetric features and cylindrical shell are probably related to collimated ejections but that occurred when the basic nebular shape had already been established.
\end{abstract}

Key words. planetary nebulae: individual: IC 5217 - ISM: jets and outflows

\section{Introduction}

The transformation of an Asymptotic Giant Branch (AGB) star into a Planetary Nebula (PN) involves a dramatic change in the mass ejection geometry. Although, in a simplified picture, it may be considered that the shell geometry changes from spherical, during the AGB phase, to axis-symmetric in the PN phase, the observations show that the mass ejection processes are much more complex. The recent detection of regularly spaced, circular thin shells in PNe indicates spherical episodic ejection of material at the very end of AGB, a fact which is not well explained yet (see Su 2004, and references therein). The presence of high velocity jets and point-symmetric structures in a noticeable fraction of PNe strongly suggests that collimated outflows or collimated winds may be common in the late AGB and/or post-AGB evolution. There is increasing evidence for PNe with multiple lobes (e.g., Sahai \& Trauger 1998) and for the coexistence of different shells with different morphology in some PNe (e.g., Benítez et al. 2005), which are difficult to explain in a classical scenario for PN formation. It is obvious that the morphological structures observed in a PN are a consequence of the physical processes that occur during the AGB to PN transition and, therefore, they contain information about these processes. Thus, a precise knowledge of these structures is a crucial step to understand PN formation.

During the last few years we have obtained images of PNe with the $1.5 \mathrm{~m}$ telescope on the Observatorio de Sierra Nevada
(Granada) to search for structures that have eluded detection in previous images. IC 5217 was included in the target list. It is a medium excitation PN (Aller \& Liller 1968) with a relatively faint $(V \simeq 15.5)$ central star that presents WR characteristics and a $T_{\text {eff }} \sim 95000 \mathrm{~K}$ (Hyung et al. 2001). After being considered as an elongated PN with the major axis along the North-South direction (Curtis 1918), the images by Balick (1987) have shown that IC 5217 presents faint open bipolar lobes oriented East-West and that the previously identified elongated structure can be better interpreted as a bright equatorial ring seen almost edge-on. The kinematics of this bright structure is compatible with an edge-on ring (Icke et al. 1989). In our images we note the existence of nebular structures that have not been reported in this PN. These structures modify drastically the ideas about IC 5217 and should be taken into account in order to understand the formation of this PN.

In this paper we present these direct images and the high resolution long-slit spectra obtained to study the internal kinematics of IC 5217. In addition, high resolution radio continuum data are also analyzed to complete our study of this PN.

\section{Observations and results}

\subsection{Optical images}

Direct images of IC 5217 were obtained during 2004 August 3 and 11 with the $1.5 \mathrm{~m}$ telescope at the Observatorio de 


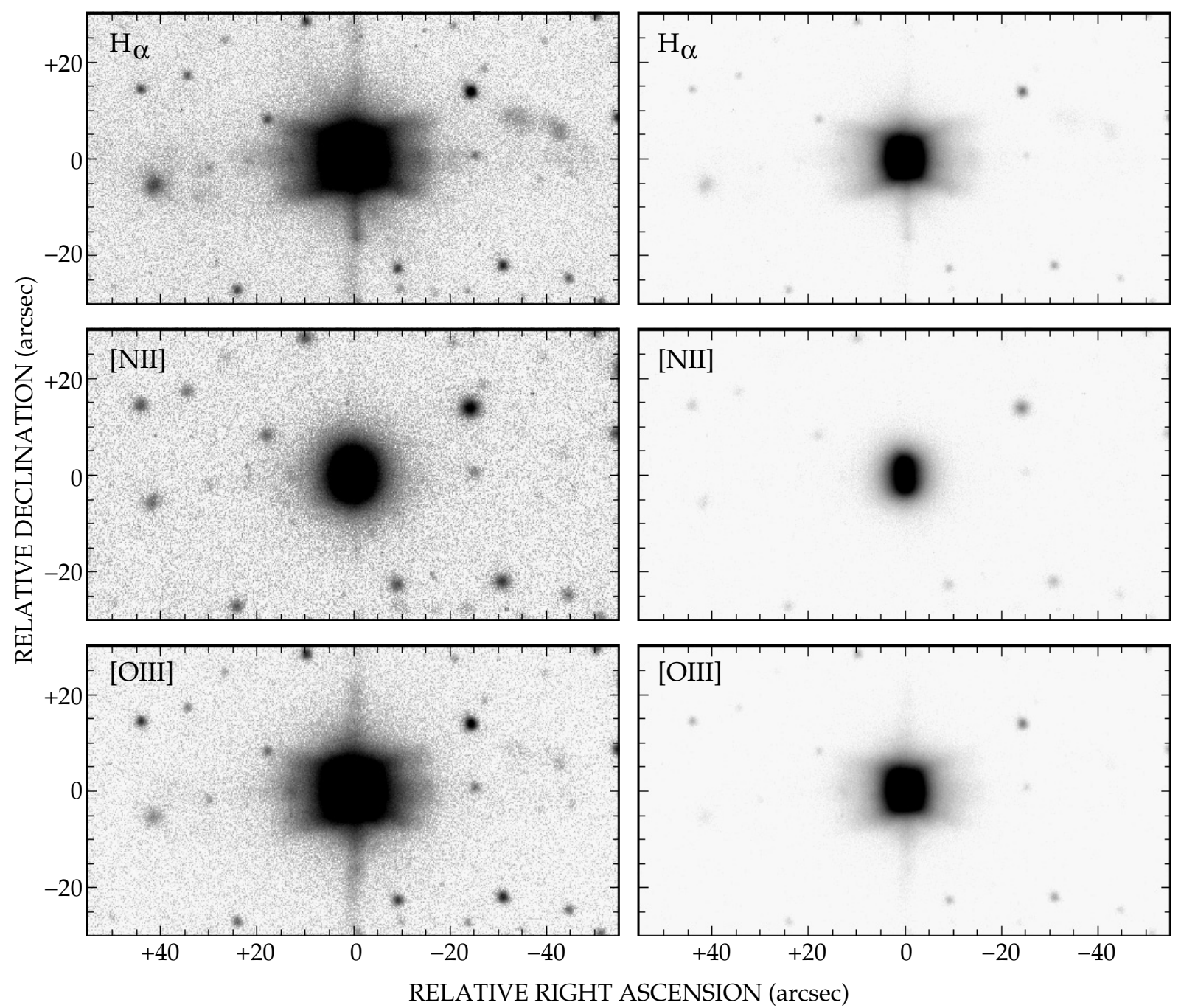

Fig. 1. Grey-scale representations of the $\mathrm{H} \alpha$, [N II] and [O III] images of IC 5217. Two different grey levels are used to highlight the different structures observed in the object. $(0,0)$ corresponds to the geometric center of the nebula (see also Fig. 2). The spatial resolution is $11^{\prime \prime} 3$ in the H $\alpha$ and $\left[\mathrm{O}\right.$ III] images and $2^{\prime \prime}$ in the [N II] one. The vertical line is an artifact due to the high brightness of the central nebular regions.

Sierra Nevada (Granada) ${ }^{1}$. The detector was a RoperScientific VersArray with $2048 \times 2048$ pixels of 0 '. 232 in size. Images were obtained through three narrow-band filters: $\mathrm{H} \alpha\left(\lambda_{\mathrm{c}}=6563 \AA\right.$, $F W H M=10 \AA),[\mathrm{N}$ II $]\left(\lambda_{\mathrm{c}}=6584 \AA, F W H M=10 \AA\right)$ and [O III] $\left(\lambda_{\mathrm{c}}=5007 \AA, F W H M=50 \AA\right)$. Exposure time was $1800 \mathrm{~s}$ for each filter. Seeing varied between $1^{\prime \prime} 3$ and $2^{\prime \prime}$ during the observations. The images were reduced following standard procedures within the MIDAS package.

Figure 1 presents grey-scale representations of IC 5217 and Fig. 2 shows contour plots of the central regions of the object. Two bright knots are observed at the center. They are oriented near the North-South direction and separated by $\simeq 2 \prime .5, \simeq 3^{\prime \prime}$ and $\simeq 2$.' 6 in $\mathrm{H} \alpha$, [N II] and [O III], respectively. These knots trace the edges of the equatorial ring. The bipolar lobes, detected in $\mathrm{H} \alpha$ and [O III] but not in [N II], depart from these knots, with an opening angle of $\simeq 80^{\circ}$ (Fig. 2). They are curved and can be traced up to $\simeq 15^{\prime \prime}$ at either side of the ring, where they are open. A limb-brightening effect is observed in the lobes. The ring and

${ }^{1}$ The Observatorio de Sierra Nevada is operated by the Consejo Superior de Investigaciones Científicas through the Instituto de Astrofísica de Andalucía (Granada, Spain). open bipolar lobes define the orientation of the major nebular axis at position angle $(\mathrm{PA}) \simeq 92^{\circ}$.

The morphology of the central bright regions observed in [N II] differs from that observed in $\mathrm{H} \alpha$ and [O III]. Whereas in $[\mathrm{N}$ II] the bright ring is the only structure detected, in $\mathrm{H} \alpha$ and [O III] the central regions present a more "squarish" shape indicating high excitation along the major nebular axis. This can be better recognized in the $\mathrm{H} \alpha /[\mathrm{N}$ II] image ratio presented in Fig. 3 that shows relatively strong $\mathrm{H} \alpha$ emission at either side (East-West) of the low excitation [N II] ring. An identical result is obtained from the $[\mathrm{O} \mathrm{III]}] /[\mathrm{N}$ II $]$ image ratio (not shown here).

A close inspection of the $\mathrm{H} \alpha$ and [O III] images reveals an elongated structure within the lobes and extending outwards. It appears as a cylindrical-like shell of $\simeq 4^{\prime \prime}$ in width, oriented at $\mathrm{PA} \simeq 87^{\circ}$. The intensity of the $\mathrm{H} \alpha$ and $[\mathrm{O} \mathrm{III}]$ emission from this structure is comparable to, and at some points, even stronger than the intensity from the edges of the open lobes and clearly dominates the emission from the central regions of the lobes (see Fig. 1). This structure can be traced up to $\sim 25^{\prime \prime}$ in the $\mathrm{H} \alpha$ image and $\sim 20^{\prime \prime}$ in [O III] one. The minimum distance is difficult to measure due to the high brightness of the central regions, but the elongated structure seems to be present at $\leq 3^{\prime \prime}$ from the center. 

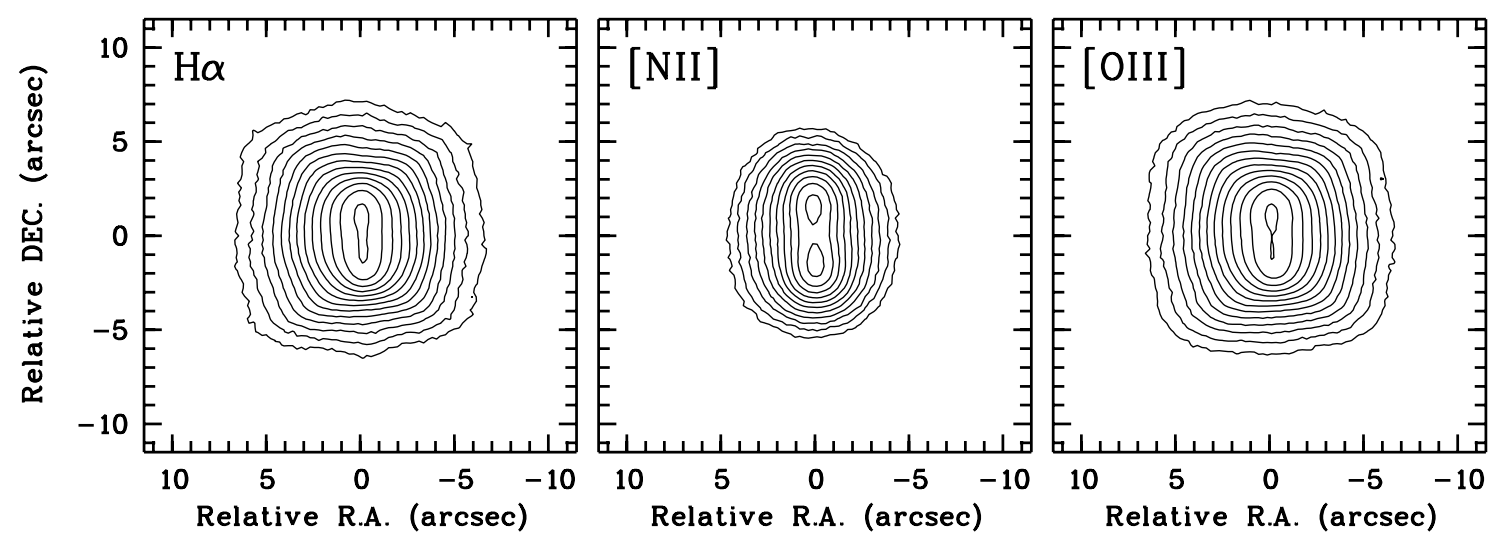

Fig. 2. Contour plots of the central regions of IC 5217 deduced from the images in Fig. 1. Contours are logarithmic separated by intensity factors of 1.6, 1.4 and 1.6 in $\mathrm{H} \alpha$, [N II] and [O III], respectively. $(0,0)$ corresponds to the geometric center of the nebula as defined by the middle point of the two observed emission peaks.

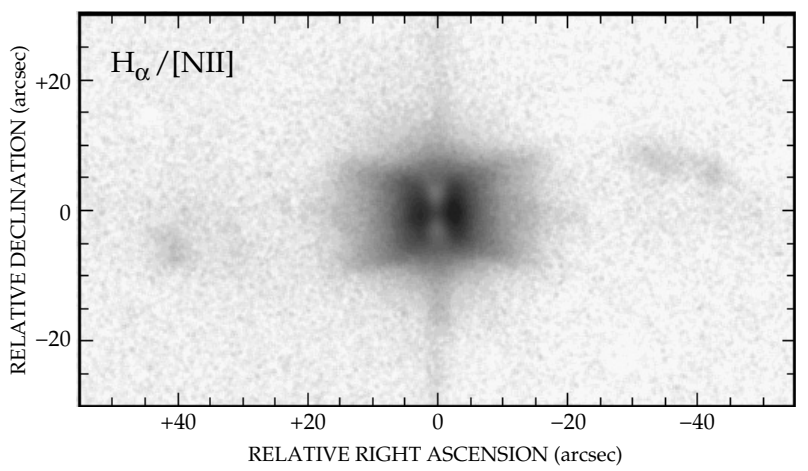

Fig. 3. Grey-scale representation of the $\mathrm{H} \alpha /[\mathrm{N}$ II] intensity ratio. Black represents high values of this ratio. $\mathrm{H} \alpha$ and $[\mathrm{N}$ II] images with the same seeing $\left(\simeq 2^{\prime \prime}\right)$ were used to obtain this image ratio.

The images also show structures at large distances from the center. In $\mathrm{H} \alpha$, faint point-symmetric features are observed at PAs $\simeq 95^{\circ}-105^{\circ}$ and $\simeq 275^{\circ}-285^{\circ}$, and at distances $\sim 32^{\prime \prime}-44^{\prime \prime}$. These features present a curvature that seems to continue that of the open lobes. In fact, extremely faint emission connects the edges of the open lobes with the point-symmetric features. Distant, faint emission is also detected at $\simeq 44^{\prime \prime}-48^{\prime \prime}$ from the center along the major nebular axis. As a whole, the $\mathrm{H} \alpha$ image strongly suggests that the open lobes may be the brighter parts of much larger point-symmetric bipolar lobes that contain the point-symmetric features and the distant faint emission. The point-symmetric features can also be recognized in [O III], although fainter than in $\mathrm{H} \alpha$. In [N II], only weak emission from some of them is detected.

\subsection{Long-slit spectroscopy}

High resolution long-slit spectra were obtained with the Manchester Echelle Spectrometer of the $2.1 \mathrm{~m}$ telescope at the San Pedro Mártir Observatory (OAN-UNAM) ${ }^{2}$ during 2004 July and 2005 August. A CCD SITe with $1024 \times 1024$ pixels was used as a detector. Slit width was $150 \mu \mathrm{m}\left(2^{\prime \prime}\right)$. The achieved spectral resolution $(F W H M)$ is $\simeq 12 \mathrm{~km} \mathrm{~s}^{-1}$. Seeing was $2^{\prime \prime}-3^{\prime \prime}$ during the observations. Table 1 present the log of the spectroscopic

${ }^{2}$ The Observatorio Astronómico Nacional is operated by the Instituto de Astronomía (UNAM) at Sierra de San Pedro Mártir (Baja California, Mexico).
Table 1. Log of spectroscopic observations.

\begin{tabular}{ccccc}
\hline \hline Slit & $\begin{array}{c}\text { PA } \\
{\left[{ }^{\circ}\right]}\end{array}$ & $\begin{array}{c}\text { Analyzed emission } \\
\text { line }\end{array}$ & Date & $\begin{array}{c}t_{\text {exp }} \\
{[\mathrm{s}]}\end{array}$ \\
\hline 1 & 0 & $\mathrm{H} \alpha$ & $2005 / 08 / 13$ & 900 \\
2 & 0 & $\mathrm{H} \alpha$ & $2005 / 08 / 13$ & 1200 \\
3 & 92 & He II 6560, [N II] 6583 & $2004 / 07 / 31$ & 1200 \\
3 & 92 & {$[\mathrm{O}$ III $] 5007$} & $2005 / 08 / 14$ & 3600 \\
4 & 2 & He II 6560, [N II] 6583 & $2004 / 07 / 28$ & 1200 \\
4 & 2 & {$[\mathrm{O}$ III $] 5007$} & $2005 / 08 / 14$ & 2700 \\
\hline \multicolumn{5}{c}{} \\
\hline
\end{tabular}

observations. The used slit positions, denoted by 1 to 4 (see Table 1), are shown in Fig. 4 overimposed on a grey-scale image of the $\mathrm{H} \alpha$ emission. The spectra were reduced following standard procedures within the IRAF package.

Figure 5 shows position-velocity (PV) maps of the [N II]6583 and [O III]5007 emission lines at Slits 3 and 4 (see Fig. 4). The detection of the [N II] emission is restricted to the equatorial ring whereas the [O III] emission is detected in an extended region.

The [N II] emission feature at Slit 3 presents two emission components located at the position of the central star and separated in velocity (as deduced from a two component Gaussian line fit) by $\simeq 54 \mathrm{~km} \mathrm{~s}^{-1}$. At Slit 4 , the $[\mathrm{N} \mathrm{II}]$ emission feature presents the shape of a velocity ellipse with a velocity splitting at the position of the central star of $\simeq 53 \mathrm{~km} \mathrm{~s}^{-1}$, and a size, at the systemic velocity, of $\simeq 3^{\prime \prime}$. 4 . Similar structures are observed in the PV maps of the [O III] emission line. In this case, the velocity splitting at the position of the central star is $\simeq 25 \mathrm{~km} \mathrm{~s}^{-1}$ and the velocity ellipse at Slit 4 has a size of $\simeq 2$ '. 6 . The He II 6560 emission feature (not shown here) presents at both slit positions a single emission peak centered at the systemic velocity and at the central star. Its velocity width $(F W H M)$ is $\simeq 20 \mathrm{~km} \mathrm{~s}^{-1}$, corrected for instrumental resolution and thermal broadening. The He II line has not been spatially resolved. The central regions of the $\mathrm{H} \alpha$ emission are saturated and no analysis can be carried out.

We note that some asymmetries exist in the [N II] velocity ellipse and in the relative intensity of the [O III] intensity maxima in the PV maps. They are probably due to small pointing and guiding errors. The PV maps at Slit 4 show that the [N II] and [O III] emissions peak at the stellar position whereas in the direct images the emissions peak in the edges of the ring. Given the used slit width and modest seeing, the position of the intensity maxima at Slit 4 could be due to components located near the 


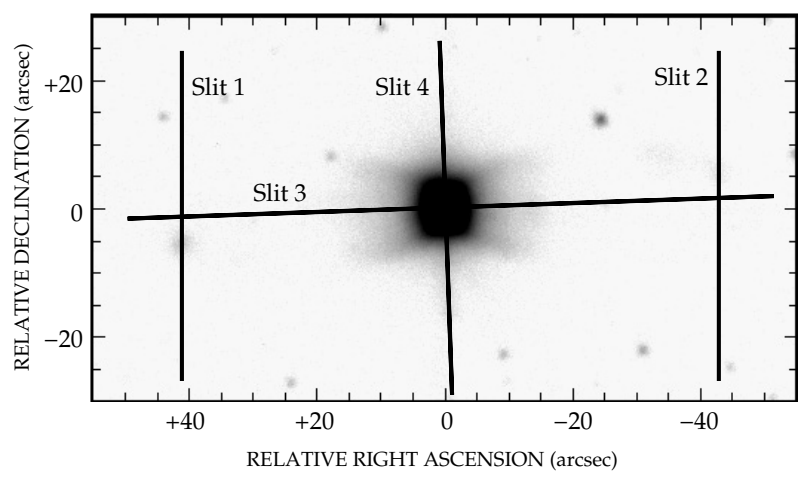

Fig. 4. Positions of the long slits used for high resolution spectroscopy overimposed on a grey-scale image of the $\mathrm{H} \alpha$ emission.

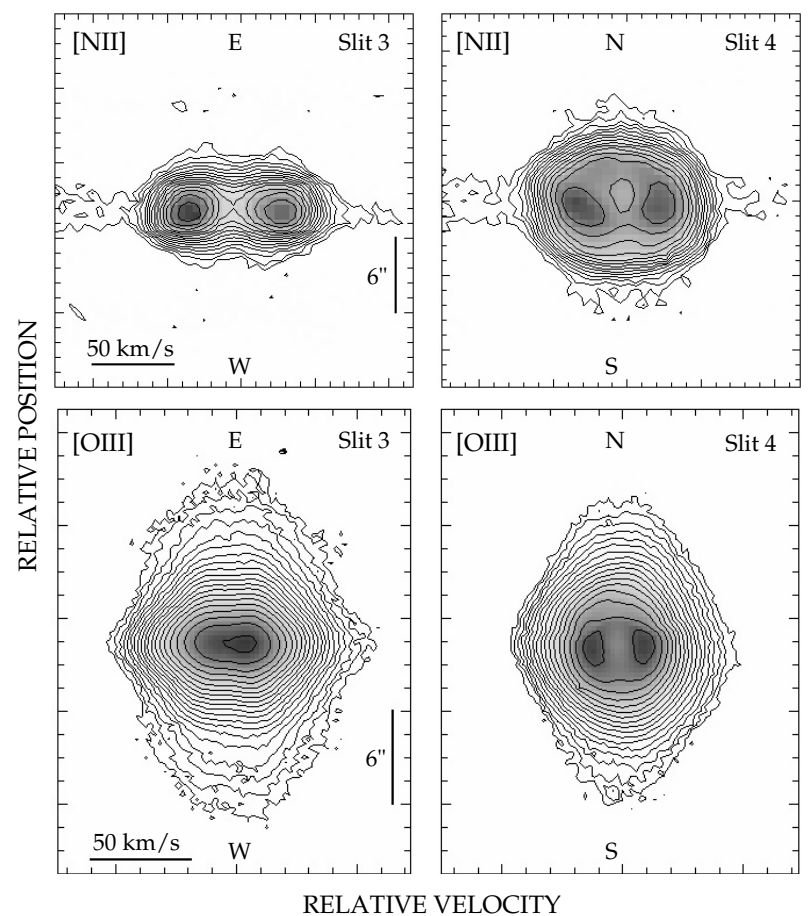

Fig. 5. Position-velocity diagrams of the [N II]6583 and [O III]5007 emission lines as observed at Slit $3\left(\mathrm{PA}=2^{\circ}\right)$ and Slit $4\left(\mathrm{PA}=92^{\circ}\right)$ (see Fig. 4). The contours are logarithmic separated by a factor 1.4 in intensity. Velocity and spatial scales are indicated. Stellar continuum is detected in the [O III] spectra but it is very faint to be seen in this representation.

ring that have not been spatially resolved (see Sect. 2.3). In any case, the size of the velocity ellipses coincides very well with the size of the ring in the images, the velocity splitting measured at both slit positions coincide with each other, and the velocity splitting measured in [N II] agrees with that obtained by Icke et al. (1987). These results indicate that the long-slit spectra at Slits 3 and 4 provide reliable spatio-kinematic information about the regions indicated in Fig. 4 and, in particular, about the ring.

The extended [O III] emission is narrower at Slit 4 than at Slit 3 (Fig. 5). Furthermore, at Slit 3 two partially resolved velocity components can be recognized, which are not identified at Slit 4. A two-component Gaussian line fit shows that the two velocity components present a constant radial velocity of $\simeq \pm 12.5 \mathrm{~km} \mathrm{~s}^{-1}$ with respect to the systemic velocity. It should be mentioned that the PV map at Slit 3 shown in Fig. 5 presents the stronger [O III] emission. At very faint intensity levels, the two velocity components can be distinguished up to a distance
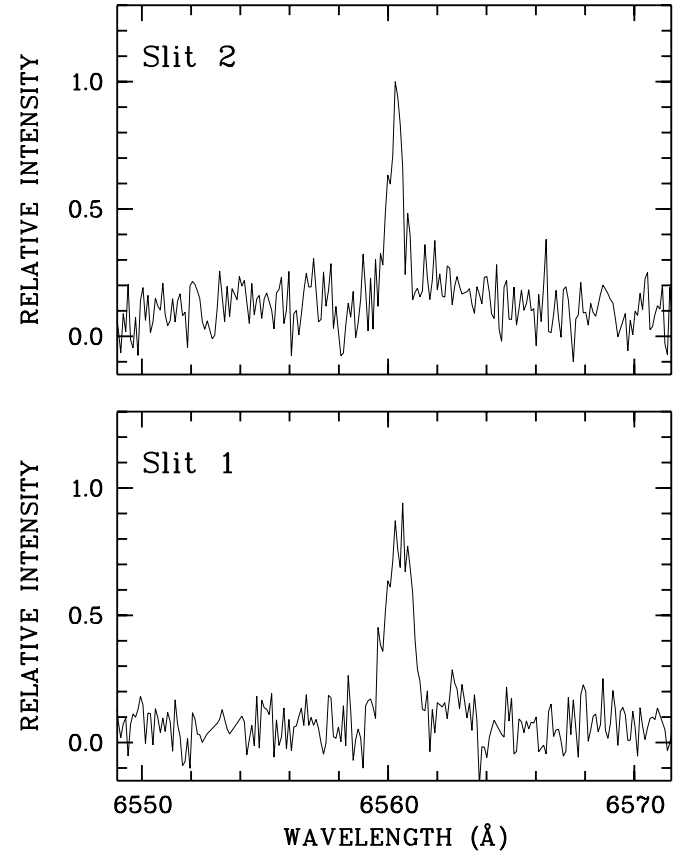

Fig. 6. Integrated spectra of the point-symmetric features, obtained at Slits 1 and 2 (see Fig. 4), around the $\mathrm{H} \alpha$ emission.

of $\sim 20^{\prime \prime}$ from the center and at the same radial velocities. The velocity separation of these two components coincides with the splitting of the [O III] emission observed at the central position.

$\mathrm{H} \alpha$ emission from the point-symmetric features has been detected at Slits 1 and 2 (see Fig. 4). Figure 6 presents the $\mathrm{H} \alpha$ line profiles obtained from these two spectra. The deduced radial velocities are $\simeq+9$ and $\simeq+15 \mathrm{~km} \mathrm{~s}^{-1}$ (with respect to the systemic velocity) for the western and eastern point-symmetric features, respectively. Given the noise of the spectra, a relatively high error $\left( \pm 5 \mathrm{~km} \mathrm{~s}^{-1}\right)$ should be considered in these radial velocities and the observed velocity difference cannot be considered significant. [N II] emission has not been detected at Slits 1 and 2, probably due the relatively short exposure time for the faintness of this emission at these positions.

\subsection{Radio continuum data}

Radio continuum observations of IC 5217 at $6 \mathrm{~cm}$ were retrieved from the VLA archive of the NRAO ${ }^{3}$. The observations (program I.D. AH509) were obtained during 1994 April 5 with the VLA in its A configuration. A bandwidth of $50 \mathrm{MHz}$ and two circular polarizations were used. The absolute flux calibrator was 3C286 (adopted flux density $7.46 \mathrm{Jy}$ at $6 \mathrm{~cm}$ ) and the phase calibrator was $1947+079$ (observed flux density $1.12 \mathrm{Jy}$ at $6 \mathrm{~cm}$ ). The on-target integration time was $117 \mathrm{~min}$. The data were processes using standard procedures within the AIPS package of the NRAO. A cleaned natural-weight map was obtained. The synthesized beam is 0 . $^{\prime} 45 \times 0.0^{\prime} 43$ at PA $5^{\circ}$ and the rms noise of the map is $14 \mu \mathrm{Jy}$, consistent with thermal noise.

Figure 7 shows the radio continuum map of IC 5217 at $6 \mathrm{~cm}$. It shows a very elongated, bright structure oriented at $\mathrm{PA} \simeq 2^{\circ}$. Obviously, the structure observed at $6 \mathrm{~cm}$ corresponds to the bright equatorial ring detected in the optical images. At $6 \mathrm{~cm}$, the size of the ring along $\mathrm{PA} \simeq 2^{\circ}$ is $\simeq 5^{\prime \prime}$ (at the $3 \sigma$ level) and its

3 The NRAO is a facility of the National Science Foundation operated under cooperative agreement by Associated Universities, Inc. 


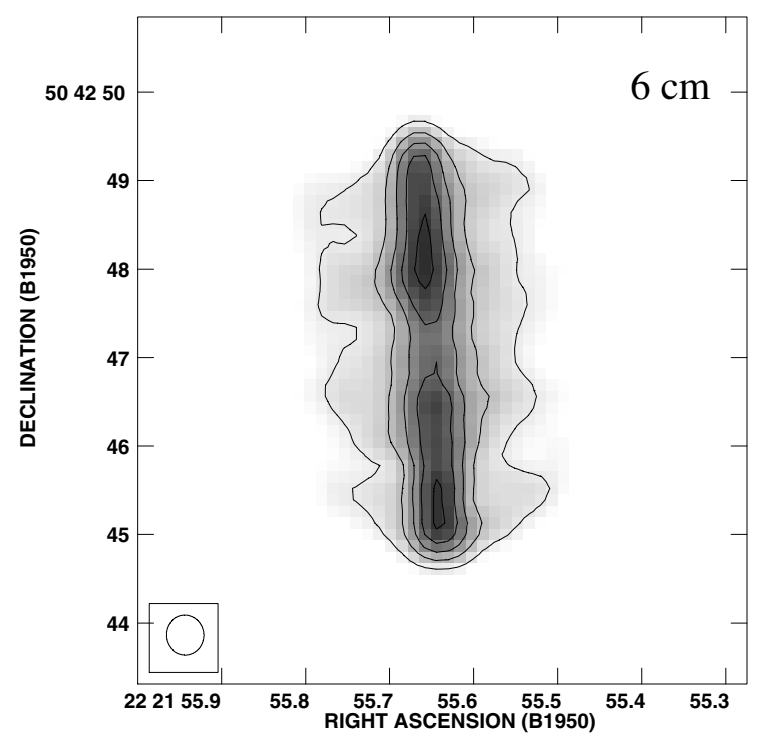

Fig. 7. Grey-scale and contour representation of the $6 \mathrm{~cm}$ continuum emission from IC 5217. The contours are $-3,3,5,7,9$ and $11 \times$ $14 \mu \mathrm{Jy}_{\text {beam }}{ }^{-1}$, the rms noise of the map. The synthesized beam, shown at the bottom left-hand corner, is $0.45 \times 0 . \prime 43$ at PA $5^{\circ}$.

deconvolved thickness along $\mathrm{PA} \simeq 92^{\circ}$ is $\simeq 0$ ' 6 . Four local emission maxima can be distinguished in the ring: two inner maxima separated by $\simeq 1^{\prime \prime} .6$ and two outer ones separated by $\simeq 3^{\prime \prime} .5$. Between the two inner maxima, a region with decreasing emission is observed, suggesting a central hole of $\sim 1^{\prime \prime}$ in diameter. Faint emission is detected at both sides of the bright ring. This emission could be related to the structures detected in the optical images (e.g., open lobes) or to represent other unresolved structures.

The flux density of IC 5217 at $6 \mathrm{~cm}$ obtained from our map is $\simeq 45.5 \mathrm{mJy}$ in agreement with the value measured by Isaacman (1984). Assuming a distance of $2.5 \mathrm{kpc}$ (Hyung et al. 2001), $T_{\mathrm{e}}=$ $10^{4} \mathrm{~K}$ and following the formulation by Mezger \& Henderson (1967) for optically thin emission, we can obtain several physical parameters for IC 5217. By considering a sphere of radius 2 '. 5 , we obtain an electron density of $\sim 3400 \mathrm{~cm}^{-3}$ and an ionized mass of $\sim 0.03 M_{\odot}$. The deduced electron density is comparable to the values obtained by Hyung et al. (2001). Given that the continuum emission in a radius of 2 .' 5 is dominated by that of the ring, the electron density and ionized mass should be considered representative of this structure.

\section{Discussion}

Our data reveal that IC 5217 contains more structural components than previously known. In the following we will try to explain the various nebular components of IC 5217 in terms of spatio-kinematic models and to use these models to discuss the possible shaping mechanism of this PN.

\subsection{The equatorial ring}

The existence of an equatorial edge-on ring in IC 5217 (Balick 1987; Icke et al. 1989) is fully confirmed by the spatio-kinematic properties of the $[\mathrm{N} \mathrm{II}]$ and [O III] emission lines in the PV maps. In particular, the presence of two velocity components at the stellar position at Slit 3 and the velocity ellipse at Slit 4 clearly indicate that the structure is a (closed) ring and that its axis is located in the plane of the sky. The $6 \mathrm{~cm}$ continuum image provides a dramatic view of the edge-on ring which appears as a very flat disk with a relatively small central hole.

From this geometry it is straightforward to obtain expansion velocities for the ring of $\simeq 27 \mathrm{~km} \mathrm{~s}^{-1}$ in $[\mathrm{N} \mathrm{II}]$ and $\simeq 12.5 \mathrm{~km} \mathrm{~s}^{-1}$ in [O III]. The expansion velocity in [N II] agrees with previous measurements (Icke et al. 1989) while the expansion velocity in [O III] is lower than the values deduced from spectrally unresolved [O III] line profiles (Sabaddin \& Hamzaoglu 1982; Robinson et al. 1982). In He II, $10 \mathrm{~km} \mathrm{~s}^{-1}$ is probably an upper limit to the expansion velocity because no velocity splitting has been detected in this emission line. Combining the expansion velocity with the radius of the ring (1'.7 in [N II] and 1'. 3 in [O III]), and assuming a distance of $2.5 \mathrm{kpc}$ (Hyung et al. 2001), kinematical ages of $\sim 750 \mathrm{yr}$ in $[\mathrm{N} \mathrm{II}]$ and $\sim 1200 \mathrm{yr}$ in [O III] are obtained.

The expansion velocity in He II and [O III] is virtually identical to the typical one of AGB envelopes $\left(10 \mathrm{~km} \mathrm{~s}^{-1}\right)$ whereas the expansion velocity in [N II] is about three times larger. Moreover, the $[\mathrm{N}$ II $]$ and $[\mathrm{O} \mathrm{III}]$ emissions show the existence of a very strong velocity gradient of $\simeq 36 \mathrm{~km} \mathrm{~s}^{-1} \operatorname{arcsec}^{-1}$ between the regions where these two emissions arise. These results indicate that the regions of the disk at a radius larger than $\sim 1$ ". 3 have been strongly accelerated while inner regions preserve the expansion velocity of the AGB envelope. The existence of this velocity gradient explains the different kinematical ages obtained from different species.

\subsection{The bipolar lobes and point-symmetric features}

According to the $\mathrm{H} \alpha$ image, the open bipolar lobes of IC 5217 may be a part of much larger bipolar lobes that also include the point-symmetric structures and distant faint regions. In a first step to analyze these structures, we will concentrate on the observed shapes.

We will consider that the open lobes can be represented by an hour-glass shape as given by

$r(\phi)=R_{\mathrm{e}}+\left(R_{\mathrm{p}}-R_{\mathrm{e}}\right) \times \sin ^{\alpha}(|\phi|)$

where $r(\phi)$ is the radius at a latitude angle $\phi$ above the equatorial plane, $R_{\mathrm{e}}$ and $R_{\mathrm{p}}$ are the equatorial and polar radius, respectively, and $\alpha$ is an exponent that permits to represent the specific hour-glass shape (see Solf \& Ulrich 1985, for details). We fix $R_{\mathrm{e}}=1$ '.25, as given by the $\mathrm{H} \alpha$ image for the equatorial ring, and vary $R_{\mathrm{p}}$ and $\alpha$ so that the curvature of the limb-brightened edges of the open lobes (within $\pm 15^{\prime \prime}$ ) is reproduced. Figure 8 shows the resulting hour-glass shape for $R_{\mathrm{p}}=46^{\prime \prime}$ and $\alpha=8$ overimposed on a contour plot of the $\mathrm{H} \alpha$ emission and we note that the results do not change very much for small variations of $R_{\mathrm{p}}$ and $\alpha$. The hour-glass shape explains the ring-open bipolar lobes system fairly well. Remarkably, the same model parameters that reproduce these two structures are also able to account for the position of the distant faint regions, that correspond to the polar regions, and the position and curvature of the pointsymmetric features, that seem to be located on the surface of large bipolar lobes. This result provides strong support for the idea that the equatorial ring, open bipolar lobes, point-symmetric features and distant faint regions represent elements of an unique entity, namely, the point-symmetric bipolar shell of IC 5217. Quantitatively, the model parameters indicate a very large axis $\left(R_{\mathrm{p}} / R_{\mathrm{e}}\right)$ ratio of $\sim 37$ and a rather moderate aspect (length-towidth) ratio of $\sim 5$ for the bipolar shell. It is obvious from these numbers that the size of the equatorial ring is comparatively extremely small and that IC 5217 can be considered as a bipolar PN with a very narrow waist. In addition, the moderate aspect ratio 


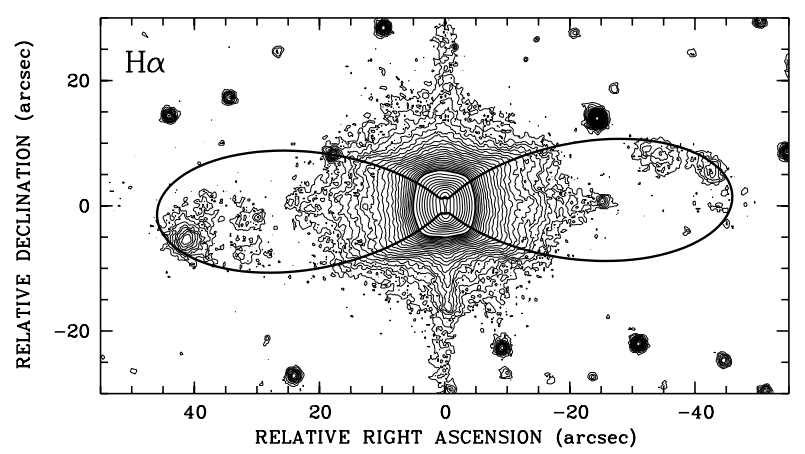

Fig. 8. Hour-glass shape (continuous line, see text) overimposed on a contour plot derived from the $\mathrm{H} \alpha$ image in Fig. 1. The contours are arbitrary and have been chosen to show the central ring, the limb-brightened edges of the bipolar lobes and the faint point-symmetric features.

indicates that the bipolar shell is not highly collimated as, e.g., in the case of M2-9 (aspect ratio 10.5, Schwarz et al. 1997).

The equatorial velocity of the bipolar shell corresponds to that of the equatorial ring $\left(12.5 \mathrm{~km} \mathrm{~s}^{-1}\right.$ in [O III]). As for the kinematics of the open lobes, the presence of two constant velocity components in the extended [O III] emission is difficult to reconcile with the observed lobe shape. The curvature of the open lobes is typical of bipolar PNe and, therefore, a continuous and smooth gradient of the radial velocity along the lobes is expected. In particular, from the shape parameters derived above and following Solf \& Ulrich (1985), one would expect an increasing from $12.5 \mathrm{~km} \mathrm{~s}^{-1}$ at the center up to $\sim 50 \mathrm{~km} \mathrm{~s}^{-1}$ at $15^{\prime \prime}$. This is not observed. Most probably, the kinematic signatures of the open lobes cannot be distinguished in the spectrum at Slit 3 because the central regions of the lobes are fainter than the elongated structure which dominates the emission along Slit 3. Lacking kinematic information, the polar expansion velocity of the bipolar shell has to be estimated using some additional hypothesis. If we assume homologous expansion (see Solf \& Ulrich 1985), and consider that the equatorial expansion velocity in $\mathrm{H} \alpha$ is similar to that in [O III], the polar expansion velocity would be $\sim 460 \mathrm{~km} \mathrm{~s}^{-1}$. This value is rather high and similar to that observed in other PNe and proto-PNe (e.g., Riera et al. 1995; Redman et al. 2000; Guerrero et al. 2004).

The low radial velocity and the hour-glass model suggest that the point-symmetric features move at right angles with respect to the observer. If so, expansion velocities of $\simeq 320-440 \mathrm{~km} \mathrm{~s}^{-1}$ are deduced assuming homologous expansion. At a distance of $2.5 \mathrm{kpc}$, a proper motion of $\simeq 0 .^{\prime} 04 \mathrm{yr}^{-1}$ is expected for the point-symmetric features, which could be detected in images obtained during the next years. In addition, the off-axis location and curvature of the point-symmetric features suggest that wobbling or precession has been involved in their formation.

\subsection{The elongated bipolar structure}

As already mentioned, on the basis of the relative intensities in the images, the extended [O III] emission in the spectrum at Slit 3 should be attributed to the elongated structure. Moreover, the presence of two constant velocity components is compatible with a cylindrical-like structure the axis of which is located in the plane of the sky and maintains this orientation all along the structure. The detection of emission very close to the ring (Fig. 5) indicates an aspect ratio of $\sim 12$ for this structure and, hence, a very high collimation degree. All these results point out that the elongated structure represents a second, highly collimated shell in IC 5217 with a cylindrical geometry. The expansion velocity law of a cylindrical shell can be described as the addition of two velocity components: a velocity component directed along the walls of the cylinder plus a velocity component perpendicular to the walls (see Guerrero et al. 2004). The spectrum at Slit 3 shows that the cross section of the cylindrical shell in IC 5217 expands at a constant velocity of $12 \mathrm{~km} \mathrm{~s}^{-1}$ whereas the velocity along the walls (perpendicular to the line of sight) should be much higher for the structure maintains its high collimation (Guerrero et al. 2004). If we assume that the (total) expansion velocity in each point of the shell is proportional to the radius from the center, an expansion velocity of $\sim 250 \mathrm{~km} \mathrm{~s}^{-1}$ is obtained at $25^{\prime \prime}$, in consonance with the expectations. A relationship between the cylindrical shell and the disk is suggested by the coincidence in radial velocity splitting in both structures. However, detailed information about the inner parts of the cylindrical shell would require optical images at higher resolution and/or deeper radio continuum images.

\subsection{The shaping of IC 5217}

Soker \& Rappaport (2000) present a list of very narrow waist bipolar PNe. A noticeable fraction of these PNe presents pointsymmetry and high expansion velocities. These properties are also found in IC 5217 which, however, differs from those PNe in some respects. Point-symmetry in most very narrow waist bipolar PNe is observed either as bright knots embedded in the shell (e.g., M 1-91) or as a large S-shaped intensity distribution in the lobes (e.g., NGC 6537). In IC 5217, the pointsymmetric structures are apparently disconnected from the open lobes which do not show point-symmetry. IC 5217 presents similarities to M 2-48 which exhibits a very narrow waist, open bipolar lobes without point-symmetry and distant collimated structures that appear disconnected from the open lobes and move at $\sim 480 \mathrm{~km} \mathrm{~s}^{-1}$ (Vázquez et al. 2000; López-Martín et al. 2002). Strong differences between IC 5217 and morphologically similar PNe are observed in the spatial distribution of the excitation. Bipolar PNe usually present prominent [N II] emission from the lobes. Point-symmetric features and jet-like components in PNe exhibit a low excitation spectrum dominated by strong [N II] emissions. In IC 5217, low excitation is observed only in the outer regions of the equatorial plane. This can be explained because IC 5217 is optically thick to the radiation of the central star only in the equatorial plane whereas it is optically thin in other directions (Icke et al. 1987; Hyung et al. 2001).

The interpretation of very narrow waist bipolar PNe presents some difficulties. Very narrow waists are not reproduced by numerical simulations of the slow wind-fast wind interaction (e.g., Frank \& Mellema 1994; Mellema \& Frank 1995). The detected equatorial disks are relatively thin and located at relatively large distances from the star to produce an effective hydrodynamical collimation (Balick 2000, and references therein). In IC 5217, the extreme flatness of the disk makes it difficult to understand how this structure can collimate the bipolar lobes and, perhaps, the cylindrical shell by purely hydrodynamical means.

Very narrow waist bipolar PNe are reproduced in scenarios involving a collimated agent (wind or magnetized jet) (Frank et al. 1998; García-Segura et al. 2005). In fact, the shape of IC 5217 compares qualitatively well with those obtained from numerical simulations by these authors. On the other hand, Soker \& Rappaport (2000) suggest a jet from an accretion disk around a companion, that compresses the slow wind from the AGB star toward the equatorial plane. In this case, a very narrow 
waist bipolar PN is expected if the PN is young and the fast wind has not smoothed out yet the original density distribution. This implies that very narrow waist bipolar PNe should be in a relatively early phase of their evolution, which is compatible with the relatively small kinematical age deduced for IC 5217 .

The point-symmetric structures or the cylindrical-like shell could represent the collimated agent. However, these structures are not perpendicular to the equatorial plane of IC 5217, as expected (Frank et al. 1998; García-Segura et al. 2005) neither perpendicular to each other. Misalignments between the collimation and disk axes may result in off-axis point-symmetric features but asymmetries in the shell could be expected (García-Segura \& López 2000). In the model by Soker \& Rappaport (2000), offaxis point-symmetric features would require a precessing accretion disk and a much more complex pattern of point-symmetric structures and/or asymmetries in the open bipolar lobes could be expected. None of these characteristics is observed in IC 5217.

Shaping the bipolar shell of IC 5217 through a collimated agent seems to require that this agent has operated along a direction perpendicular to the very narrow waist. The very faint polar regions could trace material swept up by the collimated agent (see Frank et al. 1998) whereas the point-symmetric features could be associated to a, perhaps late, manifestation of the collimated agent accompanied of wobbling/precession of the collimation axis, and the collimated agent impacts on regions near the pole, making them brighter. At this time, however, the collimated agent does not play any role in the basic shaping of the nebula, which was established before. As for the cylindrical shell, the constant orientation of its axis suggests that wobbling/precession of the collimation axis was not active during its formation or that the cylindrical shell was formed in a very short time span as in an explosive event (see Guerrero et al. 2004).

\section{Conclusions}

Optical and radio continuum images and optical high resolution, long-slit spectra of IC 5217 have revealed new aspects of this PN. In addition to the equatorial ring-open bipolar lobes system previously known, distant off-axis point-symmetric features, distant faint emission and an off-axis elongated bipolar structure have been detected in this nebula. The analysis of the data strongly suggests that the equatorial ring, open bipolar lobes, point-symmetric features and distant faint regions are elements of a single, point-symmetric bipolar shell with a very narrow waist, characterized by a very large axis ratio of $\sim 37$ and a moderate aspect ratio of $\sim 5$. Expansion velocities of $\sim 320-460 \mathrm{~km} \mathrm{~s}^{-1}$ are predicted for the polar regions and point-symmetric features. The radio continuum data shows that the equatorial ring is an extremely flat disk with a central hole. Strong acceleration of the outer regions of the disk is observed. The elongated structure presents a large aspect ratio $(\sim 12)$, probably high expansion velocities and the data are compatible with a highly collimated cylindrical shell.

The formation of the bipolar shell of IC 5217 is discussed within models that propose a collimated agent (wind or a jet) as the shaping mechanism of very narrow waist bipolar PNe. A good qualitative agreement is found between the models and the observations if the collimated agent operates in the direction perpendicular to the equatorial waist. The off-axis pointsymmetric features and cylindrical shell are probably related to collimated ejections but without being related to the main shaping of the bipolar shell.

Acknowledgements. We thank our referee (F. Sabbadin) for useful comments that have improved the interpretation of the data. We are grateful to the staffs of the Observatorio de Sierra Nevada and Observatorio de San Pedro Mártir for their assistance during the observations. We thank J. F. Gómez and M. A. Guerrero for comments and suggestions. This paper has been supported partially by grants AYA2002-00376 and AYA2005-01495 of the Spanish MEC (co-funded with Feder Funds) and PAPIIT-DGAPA-UNAM grant IN111903-3 and CONACYT grant 45848 (Mexico).

\section{References}

Aller, L. H., \& Liller, W. 1968, in Nebulae and Interstellar Matter, ed. B. M. Middlehurst, \& L. H. Aller (Chicago: Chicago University Press), 498

Balick, B. 1987, AJ, 94, 671

Balick, B. 2000, in Asymmetrical Planetary Nebulae II: From Origins to Microstructures, ed. J. H. Kastner, N. Soker, \& S. Rappaport (San Francisco: ASP Conf. Ser.), 199, 41

Benítez, G., Vázquez, R., Zavala, S., et al. 2005, in Planetary Nebulae as Astronomical Tools, ed. R. Szczerba, G. Stasińska, \& S. K. Górny (New York: AIP Conf. Proc.), 804, 64

Curtis, H. D. 1918, Publ. Lick Obs., 13, 55

Frank, A., \& Mellema, G. 1994, ApJ, 430, 800

Frank, A., Ryu, D., \& Davidson, K. 1998, ApJ, 500, 291

García-Segura, G., \& López, J. A. 2000, ApJ, 544, 336

García-Segura, G., López, J. A., \& Franco, J. 2005, ApJ, 618, 919

Guerrero, M. A., Chu, Y.-H., \& Miranda, L. F. 2004, AJ, 128, 1694

Hyung, S., Aller, L. H., Feibelman, W. A., \& Lee, W.-B. 2001, AJ, 122, 954

Icke, V., Preston, H. L., \& Balick, B. 1989, AJ, 97, 462

Isaacman, R. 1984, MNRAS, 208, 399

López-Martín, L., López, J. A., Esteban, C., et al. 2002, A\&A, 388, 652

Mellema, G., \& Frank, A. 1995, MNRAS, 273, 40

Mezger, P. G., \& Henderson, A. P. 1967, ApJ, 147, 471

Redman, M. P., O'Connor, J. A., Holloway, A. J., Bryce, M., \& Meaburn, J. 2000, MNRAS, 302, L23

Riera, A., García-Lario, P., Manchado, A., Pottasch, S. R., \& Raga, A. C. 1995, A\&A, 302, 137

Robinson, G. J., Reay, N. K., \& Atherton, P. D. 1982, MNRAS, 199, 649

Sabbadin, F., \& Hamzaoglu, E. 1982, A\&A, 110, 105

Sahai, R., \& Trauger, J. T. 1998, AJ, 116, 1357

Schwarz, H. E., Aspin, C., Corradi, R. L. M., \& Reipurth, B. 1997, A\&A, 319, 267

Soker, N., \& Rappaport, S. 2000, ApJ, 538, 241

Solf, J., \& Ulrich, H. 1985, A\&A, 148, 274

Su, K. Y. L. 2004, in Asymmetrical Planetary Nebulae III: Winds, Structure and the Thunderbird, ed. M. Meixner, J. H. Kastner, B. Balick, \& N. Soker (San Francisco: ASP Conf. Proc.), 313, 247

Vázquez, R., López-Martín, L., Miranda, L. F., et al. 2000, A\&A, 357, 1031 